\title{
Liquid salt transport growth of single crystals of the layered dichalcogenides $\mathrm{MoS}_{2}$ and $\mathrm{WS}_{2}$
}

F. Alex Cevallos ${ }^{1}$, Shu Guo ${ }^{1}$, Hoseok Heo², Giovanni Scuri², You Zhou², Jiho Sung'2, Takashi

Taniguchi ${ }^{3}$,Kenji Watanabe ${ }^{3}$, Philip Kim ${ }^{4}$, Hongkun Park ${ }^{2}$, and Robert J. Caval

${ }^{1}$ Department of Chemistry, Princeton University, Princeton NJ 08542

${ }^{2}$ Department of Chemistry and Chemical Biology, Harvard University, Cambridge MA 02138

${ }^{3}$ National Institute for Materials Science, 1-1 Namiki, Tsukuba 305-0044, Japan

${ }^{4}$ Department of Physics, Harvard University, Cambridge MA 02138 
Table S1. Crystal data and ambient temperature crystal structure refinements for $\mathrm{MoS}_{2}$.

\begin{tabular}{|c|c|}
\hline Formula & $\mathrm{MoS}_{2}$ \\
\hline formula mass(amu) & 160.06 \\
\hline crystal system & hexagonal \\
\hline space group & $P 6_{3} / m m c$ \\
\hline$a(\AA)$ & $3.1601(10)$ \\
\hline$c(\AA)$ & $12.288(4)$ \\
\hline$V\left(\AA^{3}\right)$ & $106.27(6)$ \\
\hline$Z$ & 2 \\
\hline$T(\mathrm{~K})$ & $300(1)$ \\
\hline$\rho($ calcd $)\left(\mathrm{g} / \mathrm{cm}^{3}\right)$ & 5.002 \\
\hline$\lambda(\AA)$ & 0.71073 \\
\hline$F(000)$ & 148 \\
\hline$\theta(\mathrm{deg})$ & $3.32-33.20$ \\
\hline \multirow[t]{3}{*}{ Index ranges } & $-4 \leq h \leq 4$ \\
\hline & $-4 \leq k \leq 4$ \\
\hline & $-18 \leq l \leq 18$ \\
\hline Cryst size $\left(\mathrm{mm}^{3}\right)$ & $0.051 \times 0.041 \times 0.02$ \\
\hline$\mu\left(\mathrm{mm}^{-1}\right)$ & 7.648 \\
\hline Final $R$ indices $\left(R_{1} / \omega R_{2}\right)$ & $0.0144 / 0.0367$ \\
\hline$R$ indices (all data) $\left(R_{1} / \omega R_{2}\right)$ & $0.0166 / 0.0369$ \\
\hline Residual electron density/ $\left(e \AA^{-3}\right)$ & $(-0.802)-0.873$ \\
\hline
\end{tabular}


Table S2. Wyckoff positions, coordinates, occupancies, and equivalent isotropic displacement parameters for $\mathrm{MoS}_{2}$.

\begin{tabular}{ccccccc}
\hline Atoms & $\begin{array}{c}\text { Wyck. } \\
\text { Site }\end{array}$ & $\mathrm{x} / \mathrm{a}$ & $\mathrm{y} / \mathrm{b}$ & $\mathrm{z} / \mathrm{c}$ & S.O.F. & $\mathrm{U}_{\mathrm{eq}}$ \\
\hline Mo1 & 2c & $1 / 3$ & $2 / 3$ & $1 / 4$ & $0.9686(14)$ & $0.00425(15)$ \\
$\mathrm{Mo} 2$ & $2 \mathrm{~b}$ & 0 & 0 & $1 / 4$ & $0.0314(14)$ & $0.00425(15)$ \\
$\mathrm{S} 1$ & $4 \mathrm{f}$ & $1 / 3$ & $2 / 3$ & $0.62278(8)$ & $0.9686(14)$ & $0.00620(19)$ \\
$\mathrm{S} 2$ & $4 \mathrm{e}$ & 0 & 0 & $0.377(2)$ & $0.0314(14)$ & $0.00620(19)$ \\
\hline
\end{tabular}


Table S3. Crystal data and ambient temperature crystal structure refinements for $\mathrm{WS}_{2}$.

\begin{tabular}{|c|c|}
\hline Formula & $\mathrm{WS}_{2}$ \\
\hline formula mass(amu) & 247.98 \\
\hline crystal system & hexagonal \\
\hline space group & $\mathrm{Pb}_{3} / \mathrm{mmc}$ \\
\hline$a(\AA)$ & $3.1599(4)$ \\
\hline$c(\AA)$ & $12.3554(17)$ \\
\hline$V\left(\AA^{3}\right)$ & $106.84(2)$ \\
\hline$Z$ & 2 \\
\hline$T(\mathrm{~K})$ & $300(1)$ \\
\hline$\rho($ calcd $)\left(\mathrm{g} / \mathrm{cm}^{3}\right)$ & 7.708 \\
\hline$\lambda(\AA)$ & 0.71073 \\
\hline$F(000)$ & 212 \\
\hline$\theta(\operatorname{deg})$ & $6.61-31.96$ \\
\hline \multirow[t]{3}{*}{ Index ranges } & $-4 \leq h \leq 4$ \\
\hline & $-4 \leq k \leq 4$ \\
\hline & $-18 \leq l \leq 18$ \\
\hline Cryst size $\left(\mathrm{mm}^{3}\right)$ & $0.04 \times 0.04 \times 0.02$ \\
\hline$\mu\left(\mathrm{mm}^{-1}\right)$ & 55.530 \\
\hline Final $R$ indices $\left(R_{1} / \omega R_{2}\right)$ & $0.0119 / 0.0247$ \\
\hline$R$ indices (all data) $\left(R_{1} / \omega R_{2}\right)$ & $0.0143 / 0.0255$ \\
\hline Residual electron density/ ( $\left(\AA^{-3}\right)$ & $(-1.709)-1.170$ \\
\hline
\end{tabular}


Table S4. Wyckoff positions, coordinates, occupancies, and equivalent isotropic displacement parameters respectively for $\mathrm{WS}_{2}$.

\begin{tabular}{ccccccc}
\hline Atoms & $\begin{array}{c}\text { Wyck. } \\
\text { Site }\end{array}$ & $\mathrm{x} / \mathrm{a}$ & $\mathrm{y} / \mathrm{b}$ & $\mathrm{z} / \mathrm{c}$ & $\mathrm{S} . \mathrm{O} . \mathrm{F}$. & $\mathrm{U}_{\text {eq }}$ \\
\hline $\mathrm{W} 1$ & $2 \mathrm{c}$ & $2 / 3$ & $1 / 3$ & $3 / 4$ & $0.9921(12)$ & $0.00616(16)$ \\
$\mathrm{W} 2$ & $2 \mathrm{~b}$ & 0 & 0 & $3 / 4$ & $0.0079(12)$ & $0.00616(16)$ \\
$\mathrm{S} 1$ & $4 \mathrm{f}$ & $1 / 3$ & $2 / 3$ & $0.62277(13)$ & 0.992 & $0.0077(3)$ \\
$\mathrm{S} 2$ & $4 \mathrm{e}$ & 0 & 0 & $0.380(13)$ & 0.0079 & $0.0077(3)$ \\
\hline
\end{tabular}




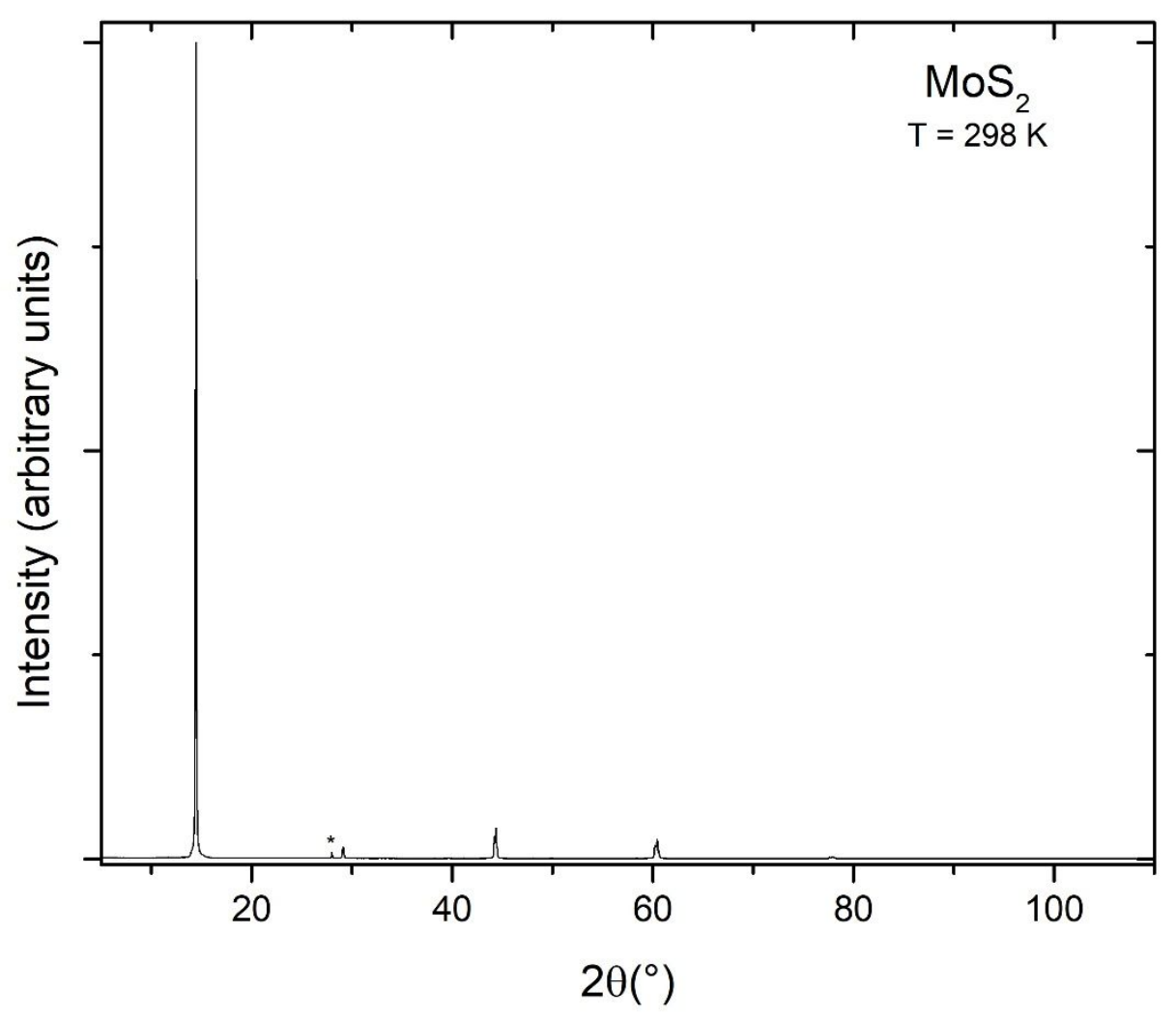

Figure S1. Powder X-ray diffraction pattern of $\mathrm{MoS}_{2}$ taken at $298 \mathrm{~K}$. The small peak indicated with an asterisk originates within the grease used to affix the sample for measurement, rather than within the sample itself. 